\title{
Segurança do paciente: estratégia de ensino-aprendizagem
}

\author{
Patient safety: teaching-learning strategy \\ Seguridad del paciente: estrategia de enseñanza-aprendizaje
}

Recebido: 23/10/2021 | Revisado: 31/10/2021 | Aceito: 02/11/2021 | Publicado: 04/11/2021

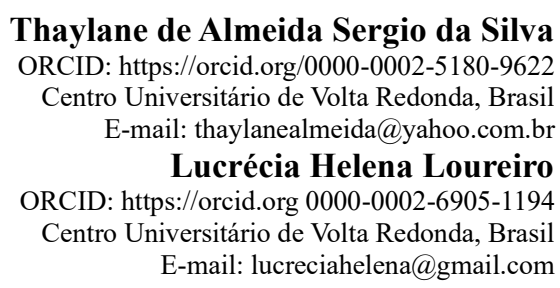

\begin{abstract}
Resumo
A segurança do paciente é uma estratégia criada para tentar diminuir os efeitos adversos na assistência e, assim, contribuir para uma qualidade segura do cuidado. O tema tem sido pauta em diversos debates e ganhado espaço nas instituições de saúde, e considerado relevante em todo o mundo por sua importância para gestores e profissionais da área de saúde no que concerne aos cuidados ao cliente e seus familiares. A estratégia está em qualificar, de maneira sólida e segura, os profissionais de saúde em fase de formação, por meio da promoção do aprendizado contínuo e organizacional, no âmbito científico e pedagógico, melhorando o desempenho de suas atividades. O objetivo deste estudo foi revisar as publicações científicas em relação às diferentes abordagens sobre segurança do paciente, principalmente a educação em saúde desses profissionais. Trata-se de um estudo quali-quantitativo utilizando os passos da revisão integrativa. Empregou-se como fonte de pesquisa o Portal de Periódicos da Capes, aplicando os descritores "Segurança do Paciente AND Enfermagem", "Segurança do Paciente AND Ensino" e "Segurança do Paciente AND Educação". Foram localizadas 72 publicações que versavam acerca do assunto, sendo selecionados 25 artigos mais relevantes relativos ao tema proposto, entre os anos de 2012 a 2017, optando-se pelas publicações nacionais. O presente estudo evidenciou a importância da segurança do paciente na formação dos profissionais de enfermagem, bem como a necessidade de uma nova abordagem no ambiente educacional para que os alunos possam ser qualificados e utilizar o conhecimento no seu ambiente profissional.
\end{abstract}

Palavras-chave: Segurança do paciente; Ensino; Enfermagem.

\begin{abstract}
Patient safety is a strategy created to try to reduce the adverse effects in care, and thus contribute to a safe quality of care. The theme has been the subject of several debates and has gained space in health institutions, and is considered relevant worldwide for its importance for managers and health professionals in terms of care for clients and their families. The strategy is to qualify, in a solid and safe way, health professionals during their training phase, by promoting continuous and organizational learning in the scientific and pedagogical scope, improving the performance of their activities. The aim of this study was to review scientific publications in relation to the different approaches to patient safety, especially the health education for these professionals. This is a qualitative and quantitative study using the steps of the integrative review. Capes Journal Portal was used as research source, applying the descriptors "Patient Safety AND Nursing", "Patient Safety AND Teaching" and "Patient Safety AND Education". There were found 72 publications that dealt with the subject, and 25 more relevant articles related to the proposed theme were selected, between the years 2012 to 2017 , and we opted for the national publications. The present study showed the importance of patient safety in the training of nursing professionals, as well as the need for a new approach in the educational environment so that the students can be qualified and use this knowledge in their professional environment.
\end{abstract}

Keywords: Patient safety; Teaching; Nursing.

\section{Resumen}

La seguridad del paciente es una estrategia creada para tratar de reducir los efectos adversos en la atención y así contribuir a una calidad de atención segura. El tema ha sido objeto de varios debates y ha ganado espacio en las instituciones de salud, y es considerado relevante a nivel mundial por su importancia para los gerentes y profesionales de la salud en cuanto a la atención a los clientes y sus familias. La estrategia es capacitar, de manera sólida y segura, a los profesionales de la salud durante su fase de formación, promoviendo el aprendizaje continuo y organizacional en el ámbito científico y pedagógico, mejorando el desempeño de sus actividades. El objetivo de este estudio fue revisar las publicaciones científicas en relación con los diferentes enfoques de la seguridad del paciente, especialmente la 
educación para la salud de estos profesionales. Este es un estudio cualitativo y cuantitativo que utiliza los pasos de la revisión integradora. Se utilizó como fuente de investigación el Portal de la Revista Capes, aplicando los descriptores "Enfermería AND Seguridad del Paciente", "Enseñanza AND Seguridad del Paciente" y "Educación AND Seguridad del Paciente". Se encontraron 72 publicaciones que abordaron el tema, y se seleccionaron 25 artículos más relevantes relacionados con la temática propuesta, entre los años 2012 a 2017, y optamos por las publicaciones nacionales. El presente estudio mostró la importancia de la seguridad del paciente en la formación de los profesionales de enfermería, así como la necesidad de un nuevo enfoque en el ámbito educativo para que los estudiantes puedan capacitarse y utilizar estos conocimientos en su entorno profesional.

Palabras clave: Seguridad del paciente; Enseñanza; Enfermería.

\section{Introdução}

A segurança do paciente está associada à proteção contra riscos, perdas e eventuais perigos. Ela é responsável por zelar pelas normas de funcionamento de determinado local e garantir que eventos adversos (EA) não ocorram.

Barbosa, Floriano, Oliveira, Nascimento e Ferreira (2016) apontam que, ao mensurar o clima de segurança de uma instituição, é possível identificar pontos fracos e fortes do comportamento dos colaboradores, além das áreas mais afetadas dentro da organização. Com essa estratégia, acredita-se que a programação das intervenções necessárias acerca da segurança poderá refletir positivamente na percepção dos profissionais no local de trabalho. Os autores ainda destacam que "as instituições de saúde têm se tornado cada vez mais conscientes da importância de se avaliar o clima de segurança, visto que é fundamental para avaliar a qualidade da assistência prestada ao paciente" (p. 2).

A preocupação com a saúde tornou-se um tema prioritário nos últimos anos, principalmente com a ocorrência de graves erros na assistência à saúde; assim, percebeu-se a necessidade de implementar protocolos para subsidiar o cuidado seguro nesta área, de modo a reduzir a ocorrência de possíveis EA no processo de cuidar (Gomes, Salvador, Rodrigues, Silva, Ferreira, \& Santos, 2017).

Dessa forma, um grande desafio para a segurança do paciente, nos serviços de saúde, é criar uma cultura de segurança que permita todas as ações da prática assistencial. Para Siman e Brito (2016), cada vez mais as organizações institucionais reconhecem a importância de se oferecerem serviços com qualidade total e redução de riscos e danos desnecessários, objetivando alcançar a satisfação e a segurança do paciente.

A assistência segura tem sido tema central de discussões na área da saúde em quase todas as partes do mundo. A Organização Mundial da Saúde (OMS) tem lançado, isoladamente ou em parceria com outras organizações, vários desafios e diretrizes com o intuito de fornecer subsídios para a discussão das realidades locais e, especialmente, para que as instituições de saúde tenham um ponto de partida para implantar e promover medidas de segurança imperativas e urgentes (Urbanetto \& Gerhardt, 2013).

Em Concepción, no Chile, a Organização Pan-Americana da Saúde criou, no ano de 2005, a Rede Internacional de Enfermagem e Segurança do Paciente e, em 2008, foi criada a Rede Brasileira de Enfermagem e Segurança do Paciente (REBRAENSP), que visa fortalecer a assistência de enfermagem segura e de qualidade (Gasparino, Bagne, Gastaldo, \& Dini, 2017).

Sobre o Programa Nacional de Segurança do Paciente (PNSP), Gomes et al. (2017) afirmam:

Em 2013, foi instituído no Brasil o Programa Nacional de Segurança do Paciente (PNSP) e, por meio dele, foram implementadas ações para a segurança do paciente em serviços de saúde, com divulgação de seis protocolos básicos voltados às áreas prioritárias, a saber: identificação do paciente; comunicação entre os profissionais de saúde; segurança na prescrição, uso e administração de medicamentos; cirurgia segura; higienização das mãos; minimização do risco de quedas e úlceras por pressão. [...] A Segurança do Paciente (SP), isto é, redução do risco de danos desnecessários associados à assistência em saúde a um mínimo aceitável. O mínimo aceitável relaciona-se às ferramentas que são viáveis diante do conhecimento atual, aos recursos disponíveis e ao contexto em que a assistência é realizada por meio 
da tomada de decisões que dizem respeito ao tratamento ou não do paciente, considerando os riscos inerentes a cada situação. (Gomes et al., 2017, p. 147)

Os EA são incidentes passíveis de ocorrer durante a prestação do cuidado à saúde e que resultam em dano ao paciente, podendo ser de natureza física, social e/ou psicológica, o que inclui doença, lesão, sofrimento, incapacidade ou morte (Gomes et al., 2017).

De acordo com Santiago e Turrini (2015), a cultura de segurança é definida como o produto de valores e padrões de comportamento individuais e de grupo, que determinam o compromisso e o estilo de gestão de uma organização. As organizações reconhecidas como detentoras de cultura de segurança positiva parecem possuir uma estrutura de comunicação franca e de confiança entre os indivíduos, mediada pelo reconhecimento da importância da segurança e da adoção de medidas preventivas no contexto organizacional. Nesse sentido, a avaliação da cultura de segurança do paciente pode ser adotada tanto para o reconhecimento da situação organizacional como para averiguar o impacto de intervenções realizadas.

Os profissionais de enfermagem são responsáveis por grande parte das ações assistenciais, portanto encontram-se em posição privilegiada para reduzir a possibilidade de incidentes que atingem o paciente, além de detectar as complicações precocemente e realizar as condutas necessárias para minimizar os danos. Uma vez que estes profissionais são os responsáveis pelo planejamento e intervenção apropriada com a finalidade de manter o ambiente seguro, é vital o desenvolvimento de pesquisa em enfermagem sobre segurança do paciente (Silva, Alves, Sanches, Terra, \& Resck, 2016).

Em vista disso, o enfermeiro deve ter uma visão ampliada do sistema de segurança do paciente e dos processos na tentativa de garantir a segurança e a qualidade do cuidado que está sob sua responsabilidade (Pinto, Schons, Busanello, \& Costa, 2015).

Parte-se do pressuposto de que o enfermeiro pode desenvolver estratégias simples e efetivas para prevenir e reduzir riscos nestes serviços, por meio do seguimento de protocolos específicos, melhores práticas associadas às barreiras de segurança nos sistemas e à educação permanente (Santiago \& Turrini, 2015).

Verifica-se, contudo, que os profissionais da equipe de enfermagem negligenciam, na maior parte das vezes, a prática de higienização das mãos, o que pode estar relacionado não diretamente à falta de conhecimento, mas à não adesão do conhecimento à prática, além da sobrecarga de tarefas, da quantidade de pacientes sob os seus cuidados e dos procedimentos realizados (Raimondi, Bernal, Souza, Oliveira, \& Matsuda, 2017).

Há evidências de que, à medida que os enfermeiros assumem seu papel de liderança junto à equipe, ocorre melhoria da assistência; por esta razão, devem relatar, comparar e mensurar os fatos e as consequências dos EA (Françolin, Gabriel, Bernardes, Silva, Brito, \& Machado, 2015).

Isto posto, o objetivo deste estudo é analisar a importância da segurança do paciente no cenário brasileiro.

\section{Metodologia}

\section{Aspectos éticos}

Por ser uma revisão integrativa, este estudo não foi submetido ao Comitê de Ética em Pesquisa. No entanto, foram mantidas as ideias dos autores das publicações utilizadas.

\section{Desenho, local do estudo, período}

Para delimitar os artigos do corpus, procedeu-se à pesquisa sistemática na base de dados informatizada oferecida pela Coordenação de Aperfeiçoamento de Pessoal de Nível Superior (Capes) - ferramenta disponível para a comunidade acadêmica brasileira -, tendo sido acessados eletronicamente no período de maio a junho de 2018. 
Trata-se de uma pesquisa descritiva/exploratória, realizada por meio de investigação bibliográfica do tipo integrativa, método de pesquisa que possibilita a síntese de estudos publicados e produz conclusões gerais a respeito de uma determinada área de pesquisa (Cestari, Florêncio, Moreira, Pessoa, Barbosa, Lima, \& Custódio, 2016).

Para elaboração da questão da pesquisa da revisão integrativa, fez-se uso da estratégia PICO: definição da questão de pesquisa, estabelecimento de critérios de inclusão e exclusão, definição das informações a serem extraídas dos estudos, avaliação dos estudos incluídos, interpretação dos resultados e síntese dos dados. Assim, a questão de pesquisa delimitada foi: "Quais são as estratégias encontradas na literatura para ensinar segurança do paciente aos profissionais de saúde?”.

\section{Amostra e critérios de inclusão e exclusão}

Optou-se pelos periódicos nacionais e restringiu-se às publicações dos últimos cinco anos (entre 2012 e 2017), tendo como descritores: "Segurança do Paciente AND Enfermagem", "Segurança do Paciente AND Ensino" e "Segurança do Paciente AND Educação". O corpus de análise foi constituído por 25 publicações, tendo sido selecionadas apenas aquelas cujo tema abordava a segurança do paciente relacionada ao ensino. Artigos em duplicata foram incluídos apenas uma vez, e aqueles cujo tema se voltava para a assistência foram descartados por não contemplarem os objetivos do presente artigo. As publicações foram selecionadas e agrupadas por conteúdos temáticos e categorias conceituais relativos à proposta deste estudo. Em seguida, foram devidamente analisadas por leitura crítica cuidadosa; e os resultados, comparados e discutidos no desenvolvimento do trabalho. O fluxograma (Figura 1) e o Quadro 1 a seguir apresentam a descrição das buscas e a seleção dos artigos:

Figura 1. Processo de seleção dos artigos desta revisão integrativa.

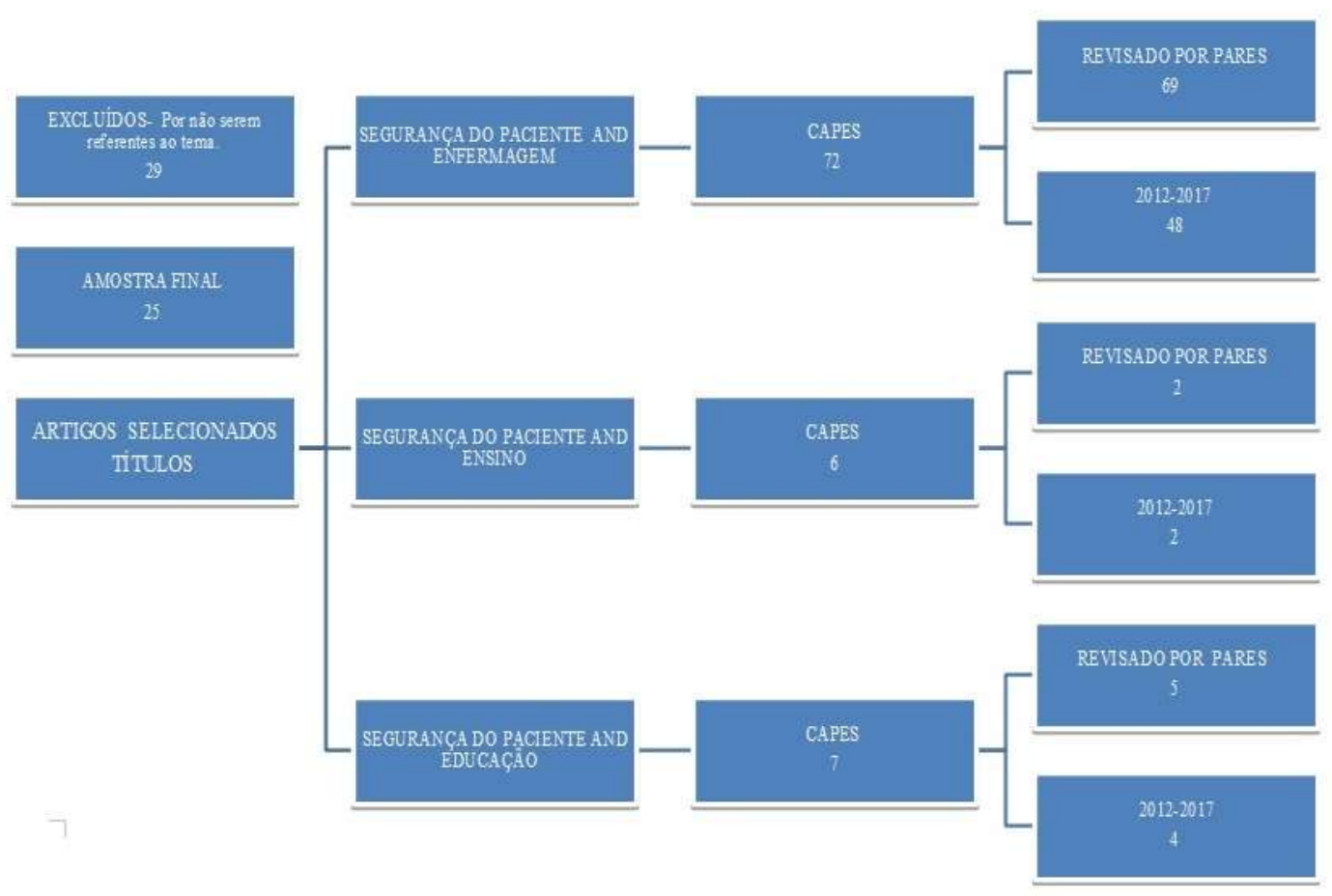

Fonte: Autores. 
Quadro 1. Descrição dos estudos incluídos na revisão integrativa, segundo título, contribuição, método, qualis, base de dados e ano de publicação.

\begin{tabular}{|c|c|c|c|c|c|c|}
\hline & TÍTULO & CONTRIBUIÇÃO & MÉTODO & $\begin{array}{l}\text { REVISTA/ } \\
\text { QUALIS }\end{array}$ & BASE/ANO & ACESSO \\
\hline A1 & $\begin{array}{l}\text { Percepção da } \\
\text { enfermagem } \\
\text { frente ao } \\
\text { clima de } \\
\text { segurança do } \\
\text { acidente em } \\
\text { instituições } \\
\text { públicas e } \\
\text { privadas }\end{array}$ & $\begin{array}{l}\text { Evidenciou que as } \\
\text { instituições privadas têm } \\
\text { demonstrado melhor } \\
\text { desempenho, e o clima de } \\
\text { segurança percebido } \\
\text { pelos profissionais foi } \\
\text { considerado } \\
\text { insatisfatório em ambas } \\
\text { as instituições }\end{array}$ & $\begin{array}{c}\text { Quantitativo/Tr } \\
\text { ansversal }\end{array}$ & $\begin{array}{c}\text { Gaúcha de } \\
\text { Enfermagem/ } \\
\text { B1 }\end{array}$ & CAPES/2018 & $\begin{array}{l}\text { www.scielo. } \\
\text { br/pdf/rgenf/ } \\
\text { v38n3/0102- } \\
\text { 6933-rgenf- } \\
\text { 38-3- } \\
\text { e68240.pdf }\end{array}$ \\
\hline A2 & $\begin{array}{l}\text { Mudanças na } \\
\text { prática de } \\
\text { enfermagem } \\
\text { para melhorar } \\
\text { a segurança } \\
\text { do paciente }\end{array}$ & $\begin{array}{l}\text { Evidenciou mudanças na } \\
\text { prática de enfermagem, } \\
\text { principalmente voltadas } \\
\text { para o gerenciamento dos } \\
\text { riscos }\end{array}$ & Qualitativo & $\begin{array}{c}\text { Gaúcha de } \\
\text { Enfermagem/ } \\
\text { B1 }\end{array}$ & CAPES/2016 & $\begin{array}{l}\text { www.seer.uf } \\
\text { rgs.br/Revist } \\
\text { aGauchadeE } \\
\text { nfermagem/a } \\
\text { rticle/view/6 } \\
8271 / 41021\end{array}$ \\
\hline A3 & $\begin{array}{c}\text { Cultura e } \\
\text { clima } \\
\text { organizacional } \\
\text { para } \\
\text { segurança do } \\
\text { paciente em } \\
\text { Unidades de } \\
\text { Terapia } \\
\text { Intensiva }\end{array}$ & $\begin{array}{l}\text { Mostrou estratégias que } \\
\text { favoreçam a } \\
\text { transferência segura de } \\
\text { informações nas trocas de } \\
\text { plantões e no manejo de } \\
\text { pacientes entre as } \\
\text { unidades }\end{array}$ & $\begin{array}{l}\text { Exploratório/Tr } \\
\text { ansversal }\end{array}$ & $\begin{array}{c}\text { Escola de } \\
\text { Enfermagem } \\
\text { da USP/A2 }\end{array}$ & CAPES/2015 & $\begin{array}{l}\text { http://www.r } \\
\text { evistas.usp.b } \\
\text { r/reeusp/artic } \\
\text { le/view/1095 } \\
\text { 48/108032 }\end{array}$ \\
\hline A4 & $\begin{array}{l}\text { Segurança do } \\
\text { paciente na } \\
\text { compreensão } \\
\text { de estudantes } \\
\text { da área da } \\
\text { saúde }\end{array}$ & $\begin{array}{llr}\text { Mostrou que } & \text { a } \\
\text { formalização do tema nos } \\
\text { diferentes níveis do } \\
\text { ensino é necessária }\end{array}$ & $\begin{array}{c}\text { Transversal/De } \\
\text { scritivo }\end{array}$ & $\begin{array}{c}\text { Gaúcha de } \\
\text { Enfermagem/ } \\
\text { B1 }\end{array}$ & CAPES/2017 & $\begin{array}{l}\text { www.seer.uf } \\
\text { rgs.br/Revist } \\
\text { aGauchadeE } \\
\text { nfermagem/a } \\
\text { rticle/view/6 } \\
\text { 4818/41698 }\end{array}$ \\
\hline A5 & $\begin{array}{c}\text { Processos de } \\
\text { medicação, } \\
\text { carga de } \\
\text { trabalho e a } \\
\text { segurança do } \\
\text { paciente em } \\
\text { unidades de } \\
\text { internação } \\
\end{array}$ & $\begin{array}{l}\text { Mostrou que o uso de } \\
\text { tecnologias, sem dúvida, } \\
\text { agrega valor para o } \\
\text { processo de cuidado } \\
\text { seguro }\end{array}$ & $\begin{array}{l}\text { Qualitativo/ } \\
\text { Descritivo }\end{array}$ & $\begin{array}{c}\text { Escola de } \\
\text { Enfermagem } \\
\text { da USP/A2 }\end{array}$ & CAPES/2015 & $\begin{array}{l}\text { https://www. } \\
\text { revistas.usp. } \\
\text { br/reeusp/arti } \\
\text { cle/view/109 } \\
\text { 521/107999 }\end{array}$ \\
\hline A6 & $\begin{array}{c}\text { A segurança } \\
\text { do paciente } \\
\text { nos caminhos } \\
\text { percorridos } \\
\text { pela } \\
\text { Enfermagem } \\
\text { Brasileira }\end{array}$ & $\begin{array}{l}\text { Verificou-se a tendência } \\
\text { para o desenvolvimento } \\
\text { de pesquisas que versam } \\
\text { sobre esta segurança no } \\
\text { cenário hospitalar, com } \\
\text { ênfase na redução do } \\
\text { risco de úlceras por } \\
\text { pressão }\end{array}$ & Quantitativo & $\begin{array}{c}\text { Revista } \\
\text { Brasileira de } \\
\text { Enfermagem/ } \\
\text { A2 }\end{array}$ & CAPES/2017 & $\begin{array}{l}\text { www.redaly } \\
\text { c.org/articul } \\
\text { o.oa?id=267 } \\
049841020\end{array}$ \\
\hline A7 & $\begin{array}{l}\text { Segurança do } \\
\text { paciente e } \\
\text { prevenção } \\
\text { cutâneo- } \\
\text { mucosas aos } \\
\text { dispositivos } \\
\text { invasivos nas } \\
\text { vias aéreas } \\
\end{array}$ & $\begin{array}{l}\text { Mostrou que os técnicos } \\
\text { de Enfermagem, atuantes } \\
\text { na UTI, apresentam } \\
\text { maior visão da clínica do } \\
\text { paciente }\end{array}$ & $\begin{array}{c}\text { Quantitativo/Q } \\
\text { ualitativo }\end{array}$ & $\begin{array}{c}\text { Escola de } \\
\text { Enfermagem } \\
\text { da USP/A2 }\end{array}$ & CAPES/2015 & $\begin{array}{l}\text { www.revista } \\
\text { s.usp.br/reeu } \\
\text { sp/article/vie } \\
\text { w/106703 }\end{array}$ \\
\hline $\mathbf{A 8}$ & $\begin{array}{l}\text { Ensino da } \\
\text { segurança do } \\
\text { paciente na } \\
\text { graduação em } \\
\text { saúde: } \\
\text { reflexões } \\
\text { sobre saberes } \\
\text { e fazeres }\end{array}$ & $\begin{array}{l}\text { Mostrou que as escolas } \\
\text { devem modificações de } \\
\text { seus } \\
\text { Pedagógicos }\end{array}$ & $\begin{array}{l}\text { Qualitativo/ } \\
\text { Descritivo }\end{array}$ & Interface/A2 & CAPES/2016 & $\begin{array}{l}\text { www.revista } \\
\text { interface.blo } \\
\text { gspot.com/2 } \\
016 / 03 /\end{array}$ \\
\hline
\end{tabular}




\begin{tabular}{|c|c|c|c|c|c|c|}
\hline A9 & $\begin{array}{l}\text { Processo de } \\
\text { contagem } \\
\text { cirúrgica: } \\
\text { evidências } \\
\text { para a } \\
\text { segurança do } \\
\text { paciente }\end{array}$ & $\begin{array}{l}\text { Infere-se a necessidade } \\
\text { de investimentos na } \\
\text { padronização } \\
\text { processo de contagem } \\
\text { cirúrgica e emprego de } \\
\text { tecnologia }\end{array}$ & $\begin{array}{l}\text { Quantitativo/Tr } \\
\text { ansversal }\end{array}$ & $\begin{array}{c}\text { Gaúcha de } \\
\text { Enfermagem/ } \\
\text { B1 }\end{array}$ & CAPES/2017 & $\begin{array}{l}\text { seer.ufrgs.br/ } \\
\text { index.php/R } \\
\text { evistaGauch } \\
\text { adeEnfermag } \\
\text { em/article/vi } \\
\text { ew/66877 }\end{array}$ \\
\hline A10 & $\begin{array}{l}\text { Educação para } \\
\text { cultura da } \\
\text { segurança do } \\
\text { paciente: } \\
\text { Implicações } \\
\text { para a } \\
\text { formação } \\
\text { profissional }\end{array}$ & $\begin{array}{l}\text { Verificou-se } \\
\text { identificação precoce do } \\
\text { erro e a tomada de } \\
\text { decisão, prevenindo a } \\
\text { ocultação e a negação das } \\
\text { equipes, bem como } \\
\text { estabelecendo estratégias } \\
\text { de prevenção de } \\
\text { recorrências das falhas } \\
\text { identificadas }\end{array}$ & $\begin{array}{l}\text { Qualitativo/ } \\
\text { Exploratório }\end{array}$ & $\begin{array}{c}\text { Revista de } \\
\text { Enfermagem } \\
\text { Anna } \\
\text { Nery/B1 }\end{array}$ & CAPES/2016 & $\begin{array}{l}\text { http://eean.e } \\
\text { du.br/default } \\
\text {.asp?ed=66 }\end{array}$ \\
\hline A11 & $\begin{array}{l}\text { Higienização } \\
\text { das mãos: } \\
\text { Adesão da } \\
\text { equipe de } \\
\text { enfermagem } \\
\text { de unidades } \\
\text { de terapia } \\
\text { intensiva } \\
\text { pediátricas }\end{array}$ & $\begin{array}{l}\text { Mostrou a não adesão } \\
\text { conhecimento à prática } \\
\text { diária, além de } \\
\text { sobrecarga de tarefas, } \\
\text { quantidade de pacientes } \\
\text { sob os seus cuidados e } \\
\text { dos procedimentos } \\
\text { realizados }\end{array}$ & $\begin{array}{l}\text { Qualitativo/ } \\
\text { Transversal }\end{array}$ & $\begin{array}{c}\text { Revista } \\
\text { Cuidarte/B2 }\end{array}$ & CAPES/2017 & $\begin{array}{l}\text { https://www. } \\
\text { revistacuidar } \\
\text { te.org/index. } \\
\text { php/cuidarte/ } \\
\text { article/view/ } \\
437\end{array}$ \\
\hline A12 & $\begin{array}{c}\text { Cultura de } \\
\text { segurança do } \\
\text { paciente em } \\
\text { instituições } \\
\text { hospitalares } \\
\text { na perspectiva } \\
\text { da } \\
\text { enfermagem }\end{array}$ & $\begin{array}{l}\text { Mostrou que o resultado } \\
\text { pode servir para o } \\
\text { planejamento e a a } \\
\text { organização das ações, } \\
\text { tendo em vista os baixos } \\
\text { escores em relação ao } \\
\text { clima de segurança, } \\
\text { gerência e percepção de } \\
\text { estresse }\end{array}$ & $\begin{array}{c}\text { Quantitativo/Tr } \\
\text { ansversal }\end{array}$ & $\begin{array}{c}\text { Gaúcha de } \\
\text { Enfermagem/ } \\
\text { B1 }\end{array}$ & CAPES/2016 & $\begin{array}{l}\text { www.seer.uf } \\
\text { rgs.br/Revist } \\
\text { aGauchadeE } \\
\text { nfermagem/a } \\
\text { rticle/downlo } \\
\text { ad/70485/40 } \\
293\end{array}$ \\
\hline A13 & $\begin{array}{l}\text { Segurança do } \\
\text { paciente na } \\
\text { práxis do } \\
\text { cuidado de } \\
\text { enfermagem: } \\
\text { Percepção de } \\
\text { enfermeiros }\end{array}$ & $\begin{array}{l}\text { Mostrou necessário } \\
\text { desenvolver movimento } \\
\text { cultural e transformador } \\
\text { na práxis do cuidado de } \\
\text { enfermagem }\end{array}$ & $\begin{array}{l}\text { Descritivo } \\
\text { /Exploratório/ } \\
\text { Qualitativo }\end{array}$ & $\begin{array}{c}\text { Revista Ibero } \\
\text { Americana/B } \\
1\end{array}$ & CAPES/2015 & $\begin{array}{l}\text { www.redaly } \\
\text { c.org/pdf/37 } \\
\text { 04/37044495 } \\
\text { 5004.pdf }\end{array}$ \\
\hline $\mathbf{A 1 4}$ & $\begin{array}{c}\text { Assistência de } \\
\text { enfermagem e } \\
\text { o enfoque da } \\
\text { segurança do } \\
\text { paciente no } \\
\text { cenário } \\
\text { brasileiro }\end{array}$ & $\begin{array}{l}\text { Mostrou a importância da } \\
\text { identificação do erro e da } \\
\text { utilização de ferramentas } \\
\text { para melhoria da cultura } \\
\text { de segurança nas } \\
\text { instituições brasileiras }\end{array}$ & $\begin{array}{l}\text { Revisão } \\
\text { Integrativa }\end{array}$ & $\begin{array}{c}\text { Saúde em } \\
\text { Debate/B2 }\end{array}$ & CAPES/2016 & $\begin{array}{l}\text { www.scielo. } \\
\text { br/scielo.php } \\
\text { ?pid=S0103- } \\
1104201600 \\
0400292 \& \text { sc } \\
\text { ript=sci...tln } \\
\text { g... }\end{array}$ \\
\hline A15 & $\begin{array}{l}\text { Percepção da } \\
\text { equipe de } \\
\text { enfermagem } \\
\text { de um hospital } \\
\text { de ensino } \\
\text { acerca da } \\
\text { segurança do } \\
\text { paciente }\end{array}$ & $\begin{array}{l}\text { Evidenciou } \\
\text { uma contemplar } \\
\text { interdisciplinar abordagem } \\
\text { transdisciplinar para o } \\
\text { desenvolvimento deste } \\
\text { tema }\end{array}$ & $\begin{array}{l}\text { Quantitativo } \\
\text { Descritivo/ } \\
\text { Exploratório }\end{array}$ & $\begin{array}{c}\text { Universidade } \\
\text { de São } \\
\text { Paulo/A2 }\end{array}$ & CAPES/2012 & $\begin{array}{l}\text { www.teses.u } \\
\text { sp.br/teses/di } \\
\text { sponiveis/7/7 } \\
\text { 140/tde- } \\
22082012- \\
154853 /\end{array}$ \\
\hline A16 & $\begin{array}{l}\text { Segurança do } \\
\text { paciente na } \\
\text { tríade } \\
\text { assistência } \\
\text { ensino } \\
\text { pesquisa }\end{array}$ & $\begin{array}{l}\text { Mostrou que os } \\
\text { educadores precisam } \\
\text { manter estratégias de } \\
\text { educação } \\
\text { permanente/continuada e } \\
\text { os projetos dos cursos } \\
\text { técnico e graduação } \\
\text { precisam } \\
\text { alinhamentos }\end{array}$ & Quantitativo & $\begin{array}{c}\text { Gaúcha de } \\
\text { Enfermagem/ } \\
\text { B1 }\end{array}$ & CAPES/2013 & $\begin{array}{l}\text { seer.ufrgs.br/ } \\
\text { RevistaGauc } \\
\text { hadeEnferma } \\
\text { gem/article/v } \\
\text { iew/43294 }\end{array}$ \\
\hline
\end{tabular}




\begin{tabular}{|c|c|c|c|c|c|c|}
\hline A17 & $\begin{array}{c}\text { Clima de } \\
\text { segurança do } \\
\text { paciente em } \\
\text { um hospital } \\
\text { privado }\end{array}$ & $\begin{array}{l}\text { Possibilitou } \\
\text { direcionamento de ações } \\
\text { que poderão ser } \\
\text { realizadas com maior } \\
\text { qualidade, e prezando } \\
\text { pela segurança do } \\
\text { profissional e do paciente }\end{array}$ & $\begin{array}{l}\text { Quantitativo } \\
\text { Descritivo } \\
\text { /Exploratório }\end{array}$ & $\begin{array}{c}\text { Texto \& } \\
\text { Contexto } \\
\text { Enfermagem/ } \\
\text { A2 }\end{array}$ & CAPES/2016 & $\begin{array}{l}\text { www.scielo. } \\
\text { br/pdf/tce/v2 } \\
\text { 5n3/pt_0104 } \\
-0707-\text { tce- } \\
\text { 25-03- } \\
\text { 1460015.pdf }\end{array}$ \\
\hline A18 & $\begin{array}{l}\text { Banho no } \\
\text { leito: Carga de } \\
\text { trabalho } \\
\text { equipe de } \\
\text { enfermagem e } \\
\text { segurança do } \\
\text { paciente } \\
\end{array}$ & $\begin{array}{l}\text { Evidenciou que a não } \\
\text { organização do cuidado } \\
\text { pode ocasionar eventos } \\
\text { adversos }\end{array}$ & $\begin{array}{c}\text { Quantitativo/Q } \\
\text { ualitativo }\end{array}$ & $\begin{array}{c}\text { Texto \& } \\
\text { Contexto } \\
\text { Enfermagem/ } \\
\text { A2 }\end{array}$ & CAPES/2015 & $\begin{array}{l}\text { www.index- } \\
\text { f.com/textoc } \\
\text { ontexto/2015 } \\
\text { /r241044.ph } \\
\text { p }\end{array}$ \\
\hline A19 & $\begin{array}{l}\text { Higienização } \\
\text { das mãos e a } \\
\text { segurança do } \\
\text { paciente: } \\
\text { Perspectiva de } \\
\text { docentes e } \\
\text { universitários } \\
\end{array}$ & $\begin{array}{l}\text { Mostrou que não há uma } \\
\text { infraestrutura para } \\
\text { práticas preconizadas de } \\
\text { higienização das mãos, } \\
\text { fato que pode contribuir } \\
\text { para a ocorrência de } \\
\text { falhas no processo }\end{array}$ & $\begin{array}{l}\text { Quantitativo/E } \\
\text { xploratório }\end{array}$ & $\begin{array}{c}\text { Texto \& } \\
\text { Contexto } \\
\text { Enfermagem/ } \\
\text { A2 }\end{array}$ & CAPES/2013 & $\begin{array}{l}\text { www.index- } \\
\text { f.com/textoc } \\
\text { ontexto/2013 } \\
\text { /r22-901.php }\end{array}$ \\
\hline A20 & $\begin{array}{c}\text { Saúde dos } \\
\text { trabalhadores } \\
\text { de } \\
\text { enfermagem e } \\
\text { a segurança } \\
\text { do paciente: O } \\
\text { olhar de } \\
\text { gerentes de } \\
\text { enfermagem }\end{array}$ & $\begin{array}{l}\text { Evidenciou um cotidiano } \\
\text { de trabalho marcado por } \\
\text { sofrimento e } \\
\text { preocupação, devido aos } \\
\text { altos índices de } \\
\text { absenteísmo decorrentes } \\
\text { do adoecimento }\end{array}$ & Qualitativo & $\begin{array}{l}\text { Revista da } \\
\text { Escola de } \\
\text { Enfermagem } \\
\text { da USP/A2 }\end{array}$ & CAPES/2015 & $\begin{array}{l}\text { https://www. } \\
\text { revistas.usp. } \\
\text { br/revusp/ind } \\
\text { ex }\end{array}$ \\
\hline A21 & $\begin{array}{l}\text { Gerenciament } \\
\text { o da } \\
\text { segurança do } \\
\text { paciente sob a } \\
\text { ótica dos } \\
\text { enfermeiros }\end{array}$ & $\begin{array}{lr}\text { As instituições } & \text { devem } \\
\text { desenvolver políticas } \\
\text { organizacionais, focadas } \\
\text { no estímulo à notificação } \\
\text { de eventos e na } \\
\text { implementação } & \text { de } \\
\text { medidas que direcionem } \\
\text { para uma cultura } \\
\text { organizacional ra não } \\
\text { punitiva }\end{array}$ & $\begin{array}{l}\text { Quantitativo/D } \\
\text { escritivo }\end{array}$ & $\begin{array}{l}\text { Revista da } \\
\text { Escola de } \\
\text { Enfermagem } \\
\text { da USP/A2 }\end{array}$ & CAPES/2015 & $\begin{array}{l}\text { https://www. } \\
\text { revistas.usp. } \\
\text { br/reeusp/arti } \\
\text { cle/view/103 } \\
197\end{array}$ \\
\hline A22 & $\begin{array}{l}\text { Compreensão } \\
\text { de alunos de } \\
\text { cursos de } \\
\text { graduação em } \\
\text { enfermagem e } \\
\text { medicina } \\
\text { sobre } \\
\text { segurança do } \\
\text { paciente }\end{array}$ & $\begin{array}{l}\text { Mostrou que os alunos } \\
\text { são capazes de relacionar } \\
\text { alguns dos aspectos } \\
\text { pesquisados sobre } \\
\text { segurança do paciente } \\
\text { com a experiência vivida } \\
\text { nos estágios curriculares }\end{array}$ & $\begin{array}{l}\text { Qualitativo/ } \\
\text { Exploratório }\end{array}$ & $\begin{array}{l}\text { Acta Paulista } \\
\text { de } \\
\text { Enfermagem/ } \\
\text { A2 }\end{array}$ & CAPES/2013 & $\begin{array}{l}\text { www.unifes } \\
\text { p.br/acta/pdf } \\
\text { /v26/n1/v26n } \\
\text { 1a5.pd }\end{array}$ \\
\hline $\mathbf{A 2 3}$ & $\begin{array}{l}\text { Educação em } \\
\text { Saúde: } \\
\text { Reflexões a } \\
\text { partir da } \\
\text { vivência de } \\
\text { Residentes } \\
\text { Multiprofissio } \\
\text { nais }\end{array}$ & $\begin{array}{l}\text { Os integrantes da } \\
\text { residência } \\
\text { multiprofissional } \\
\text { relataram a importância } \\
\text { dessas atividades na } \\
\text { política de segurança do } \\
\text { paciente, na formação } \\
\text { profissional e na } \\
\text { formação do vínculo } \\
\text { entre profissional e } \\
\text { usuário }\end{array}$ & Quantitativo & $\begin{array}{c}\text { Tempus } \\
\text { Actas de } \\
\text { Saúde } \\
\text { Coletiva/B3 }\end{array}$ & CAPES/2017 & $\begin{array}{l}\text { www.tempus } \\
\text { actas.unb.br > } \\
\text { Capa > v. 10, } \\
\text { n. } 4(2016)> \\
\text { S }\end{array}$ \\
\hline A24 & $\begin{array}{l}\text { Educação do } \\
\text { paciente sobre } \\
\text { regime } \\
\text { terapêutico } \\
\text { medicamentos } \\
\text { o no processo } \\
\text { de alta } \\
\text { hospitalar: }\end{array}$ & $\begin{array}{lr}\text { Mostrou a educação do } \\
\text { paciente sobre } & \text { regime } \\
\text { terapêutico } & \\
\text { medicamentos } & \text { no } \\
\text { processo de alta } \\
\text { hospitalar }\end{array}$ & $\begin{array}{l}\text { Qualitativo/ } \\
\text { Integrativo }\end{array}$ & $\begin{array}{c}\text { Gaúcha de } \\
\text { Enfermagem/ } \\
\text { B1 }\end{array}$ & CAPES/2013 & $\begin{array}{l}\text { eer.ufrgs.br/ } \\
\text { RevistaGauc } \\
\text { hadeEnferma } \\
\text { gem/article/v } \\
\text { iew/20885 }\end{array}$ \\
\hline
\end{tabular}


Research, Society and Development, v. 10, n. 14, e348101422199, 2021

(CC BY 4.0) | ISSN 2525-3409 | DOI: http://dx.doi.org/10.33448/rsd-v10i14.22199

\begin{tabular}{|c|c|c|c|c|c|c|}
\hline & $\begin{array}{l}\text { Uma revisão } \\
\text { integrativa }\end{array}$ & & & & & \\
\hline A25 & $\begin{array}{l}\text { Cultura da } \\
\text { segurança do } \\
\text { paciente na } \\
\text { atenção } \\
\text { primária à } \\
\text { saúde }\end{array}$ & $\begin{array}{l}\text { Mostrou a necessidade do } \\
\text { trabalho em equipe com } \\
\text { uma adequada } \\
\text { comunicação entre os } \\
\text { profissionais }\end{array}$ & Qualitativo & $\begin{array}{c}\text { Texto \& } \\
\text { Contexto } \\
\text { Enfermagem/ } \\
\text { A2 }\end{array}$ & CAPES/2013 & $\begin{array}{l}\text { https://revist } \\
\text { as.ufpr.br/co } \\
\text { gitare/article/ } \\
\text { download/45 } \\
665 / 2852\end{array}$ \\
\hline
\end{tabular}

Fonte: Autores.

\section{Resultados e Discussão}

Na presente revisão integrativa, 25 (100 \%) publicações são do Portal de Periódicos da Capes. Foi publicado um artigo no ano de 2010; um em 2012; cinco em 2013; seis em 2015; cinco em 2016 e 2017; um em 2018. No que se refere à qualificação da revista, 13 (52\%) correspondem a Qualis A2; nove (36\%) a Qualis B1; dois (8\%) a Qualis B2; e um (4\%) a Qualis B3, conforme demonstra o Quadro 2. 
Quadro 2. Síntese das unidades de registro e significados para análise.

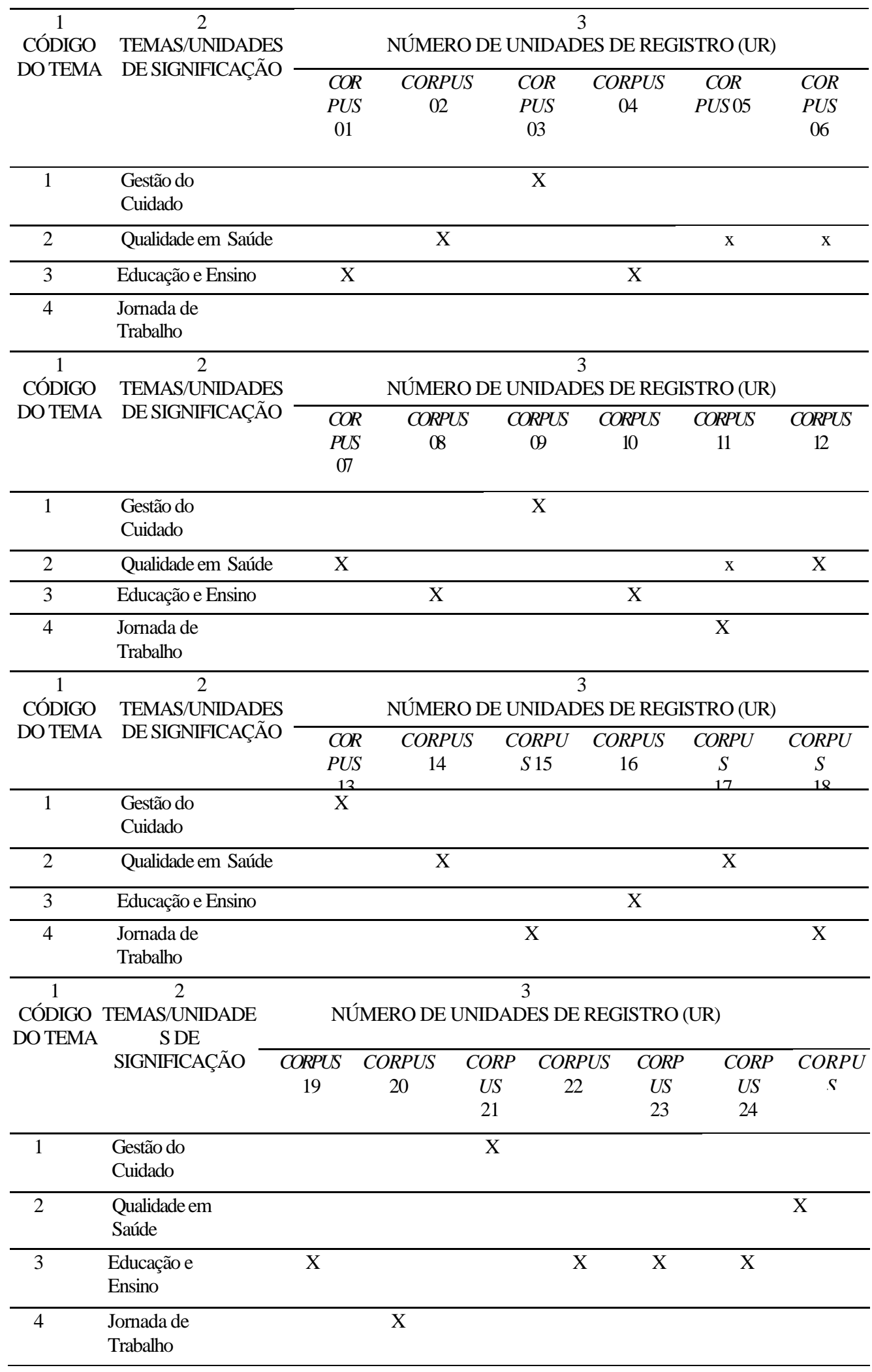

Fonte: Autores.

Após leitura dos resumos, 25 artigos foram selecionados, analisados e compuseram o corpus da pesquisa por abordarem a segurança do paciente e terem como foco a equipe de enfermagem e as estratégias adotadas pelos enfermeiros para inserir o tema nas instituições observadas. 
No que tange à revisão integrativa, esta auxilia o conhecimento atual sobre o tema pesquisado, pois visa analisar, interpretar e sintetizar os resultados do estudo, contribuindo para um pensamento crítico. Desse modo, os artigos foram agrupados em três categorias: 1) Educação e Ensino, 2) Gestão do Cuidado e 3) Jornada de Trabalho.

\section{Categoria 1: Educação e Ensino}

De acordo com os 25 artigos analisados, nove (36\%) destacaram a importância da educação e ensino.

A formação acadêmica dos profissionais de saúde, seja nas universidades ou em cursos técnicos, reforça a premissa do desenvolvimento do trabalho sem erros. Os autores apontam que são expressamente inaceitáveis a ocorrência de erros relacionados à falta de cuidado, conhecimento e atenção por parte desses profissionais, que têm a responsabilidade de salvar vidas. (Yoshikawa, Sousa, Peterlini, Kusahara, Pedreira, \& Avelar, 2013, p. 22)

Nesse contexto, é importante frisar a importância de as universidades inserirem em seus conteúdos programáticos a temática segurança do paciente para os alunos da área da saúde. Diante do exposto, a Organização Mundial de Saúde (OMS) desenvolveu um guia multiprofissional para organização do currículo de segurança do paciente, com objetivo de auxiliar as instituições acadêmicas de saúde na formação de profissionais nessa área (Marra \& Sette, 2016).

Por outro lado, o Programa Nacional de Segurança do Paciente (PNSP), lançado em 2013 pelo Ministério da Saúde (MS), reforça esta premissa, pois possui como um dos seus objetivos fomentar a inclusão do tema segurança do paciente no ensino técnico, na graduação e na pós-graduação na área da saúde. (Cauduro, Magnago, Andolhe, Lanes, \& Ongaro, 2017, p. 2)

A fim de garantir que a prática da segurança efetivamente aconteça, o Conselho Federal de Enfermagem (Cofen) e o Conselho Regional de Enfermagem (Coren) criaram um selo de qualidade e certificação para os profissionais de enfermagem e instituições como forma de reconhecer o esforço de todos e, principalmente, motivar toda a equipe a trabalhar com esse olhar (Conselho Federal de Enfermagem [Cofen], 2018).

O selo de qualidade conferido pelos conselhos de classe da enfermagem contemplará as instituições de saúde e de ensino e estará voltado para duas áreas prioritárias, que são a segurança do paciente e a gestão de risco, conforme descreve o presidente do conselho: "Entendemos que a formação profissional é o berço de todo o processo de exercício profissional e, por isto, também poderá passar pelo processo de certificação da qualidade" (Cofen, 2018, p. 2).

Segundo o Cofen (2018), "o programa de qualidade traz a possibilidade de certificar unidades institucionais, reconhecendo o esforço e a excelência daquele serviço, ainda que a instituição como um todo ainda não esteja apta à certificação" (p. 1). O conselho ainda determina:

Para conseguir a certificação, as instituições serão avaliadas em seis dimensões: "Ações gerenciais sistêmicas", "Estrutura organizacional", "Aspectos operacionais", "Infraestrutura", "Gestão de pessoas" e "Responsabilidade social". O selo de qualidade é uma certificação dos profissionais de Enfermagem e não apenas das instituições. (Cofen, 2018, p. 1)

Em vista disso, ao trabalhar as seis dimensões com os acadêmicos e estudantes de enfermagem, será possível estabelecer a cultura de segurança do paciente e atitudes efetivas para um cuidado seguro nos estabelecimentos de saúde.

Para Cauduro et al. (2017), o processo de formação dos futuros profissionais de saúde deve contemplar conhecimentos técnico-científicos, que os tornem capazes de intervir no processo saúde/doença, por meio de ferramentas que garantam a qualidade da assistência à saúde. Uma delas, utilizada atualmente, é o Protocolo Nacional de Segurança do Paciente (PNSP), que enumera as diretrizes e protocolos de segurança para o desenvolvimento de práticas seguras. 
A estratégia mais comumente empregada para implantar a cultura de segurança do paciente nos estabelecimentos de saúde é a educação continuada. Com o intuito de estabelecer essa cultura, Wegner, Silva, Kantorski, Predebon, Sanches e Pedro (2016) mencionam que os serviços estão oferecendo treinamentos, palestras, cursos e aulas teóricas aos profissionais como tática para apresentar a temática aos seus colaboradores.

Sendo assim, acredita-se que o treinamento das equipes possibilite a revisão de conteúdos abordados anteriormente, assim como esclarecimentos de possíveis dúvidas dos profissionais, além de auxiliar nas orientações para os acompanhantes e/ou cuidadores.

Apontam Teixeira, Rodrigues e Machado (2012) que é fundamental a compreensão do paciente, familiar e/ou cuidador acerca da segurança do paciente e que essa orientação deve estar pautada nas diretrizes da OMS. É, também, essencial verificar se as orientações fornecidas foram absorvidas pelos colaboradores, solicitando a eles que expliquem o plano com suas próprias palavras.

Além disso, é importante instituir programação de seguimento pós-alta; conciliação do plano terapêutico medicamentoso; revisão sobre como proceder na ocorrência de um problema, orientando sobre qual serviço de atendimento deve procurar; além de fornecer instruções por escrito. (Teixeira et al., 2012, p. 189)

Ademais, a educação permanente em saúde incentiva a diversificação de estratégias para compartilhar conhecimentos e experiências do mundo do trabalho. Wegner et al. (2016) esclarecem que o profissional da saúde que discute e aceita a possibilidade da ocorrência de eventos adversos (EA) está progredindo para uma cultura de segurança do paciente.

Cabe ressaltar, ainda, a importância do enfermeiro para as orientações no momento da alta hospitalar, por meio do aconselhamento e, principalmente, do esclarecimento das dúvidas da família e/ou cuidador, garantindo, desta forma, continuidade na qualidade da assistência.

\section{Categoria 2: Gestão do Cuidado}

De acordo com os artigos analisados, 12 (48\%) estão relacionados à gestão do cuidado e aos procedimentos seguros e adequados às necessidades dos pacientes hospitalizados.

Para a implantação e incorporação da cultura de segurança do paciente em qualquer instituição de saúde, faz-se necessário elevado nível de comprometimento da gerência e dos profissionais da instituição, bem como forte espírito de coesão entre os diversos departamentos, fatores imprescindíveis para a garantia de assistência segura, tanto para o profissional como para o paciente (Françolin et al., 2015).

A segurança do paciente se caracteriza pela redução de riscos e erros cometidos pelos profissionais de enfermagem e por qualquer profissional que esteja praticando a assistência, sendo responsabilidade integral do cuidado. Desse modo, o enfermeiro pode ser um facilitador no processo de identificação dos eventos adversos (EA).

Nesse sentido, no ano de 2009, foram divulgadas as metas internacionais e diretrizes para segurança do paciente: identificação correta do paciente; comunicação clara e efetiva; segurança na administração de medicamentos; maior segurança em cirurgias, diminuição dos riscos de infecção e queda do paciente (Gasparino et al., 2017).

Françolin et al. (2015), em seu estudo, enfatizam que a ocorrência de EA tem diminuído significativamente quando o enfermeiro está nas comissões de gerenciamento de riscos. Perceberam-se também as mudanças positivas decorrentes das ações propostas por esse profissional.

Vale destacar que o enfermeiro é responsável técnico pela equipe de enfermagem frente ao seu conselho de classe, e irá responder a possíveis processos judiciais relacionados à ocorrência desses eventos. 
Por este motivo, devem relatar os casos de EA, comparar e mensurar os fatos e suas consequências, e assim conscientizar a equipe da importância de seguir as diretrizes de segurança do paciente no ambiente de trabalho.

Uma das principais repercussões da segurança do paciente nos estabelecimentos hospitalares é a compreensão, junto à equipe de saúde, da importância de potencializar mudanças, assimilando a cultura de segurança como um processo de socialização que permite o (re)pensar na assistência à saúde, tanto na perspectiva de educação permanente aos profissionais atuantes no sistema, como de transformação de tais cenários de ensino-aprendizagem para futuros profissionais. No entanto, compreende-se que criar/transformar uma cultura pode ser um processo longo (porém necessário e possível), no que concerne à qualidade do cuidado ao paciente (Cauduro et al., 2017).

\section{Categoria 3: Jornada de Trabalho}

De acordo com os artigos analisados, quatro (16\%) enfatizaram a jornada de trabalho como um desafio para a segurança do paciente. Existe crescente evidência de que a carga de trabalho da equipe de enfermagem está associada com a segurança do paciente em hospitais, contudo ainda persistem várias lacunas de conhecimento sobre a natureza da relação entre a carga de trabalho da equipe de enfermagem e a segurança dos pacientes (Magalhães, Moura, Pasin, Funcke, Pardal, \& Kreling, 2015).

Corroborando esta assertiva, Möller e Magalhães (2015) salientam que, entre as barreiras que influenciam no descumprimento das precauções-padrão pela equipe de enfermagem, destacam-se a sobrecarga de trabalho, a falta de pessoal e o tempo de experiência profissional.

Além da relação com a quantidade de profissionais de enfermagem disponíveis, também se observou a relevância dos ambientes de trabalho que promovem processos organizacionais complexos e descontinuados, os quais favorecem o estresse e a desconcentração dos profissionais, bem como adaptações às normas de segurança com vistas a facilitar o serviço. Por este motivo, envolvem diariamente riscos potenciais (Möller \& Magalhães, 2015).

No contexto brasileiro, o ambiente e o sistema de atendimento afetam as práticas de enfermagem, de modo que alguns problemas relativos à falta de estrutura e materiais para atender os pacientes aparecem como adversidades no ambiente de trabalho das instituições de saúde.

Desse modo, situações simultâneas e inesperadas, assim como questões de estrutura física, tornam os ambientes de trabalho desfavoráveis, complicando o exercício da atenção qualificada e segura pela enfermagem.

O estudo de Baptista, Pustiglione, Almeida, Felli, Garzin e Melleiro (2015) revelou que as cargas e os desgastes ocorridos entre os trabalhadores de enfermagem de um hospital universitário brasileiro despontaram afastamentos em períodos inferiores a 15 dias, de forma recorrente, frequentemente pelos mesmos motivos, e que esses afastamentos de curto período, quando analisados ao longo de 12 meses, representaram uma quantidade significativa de dias perdidos de trabalho.

Este fato parece indicar que os afastamentos impactam não somente a saúde do trabalhador, mas também geram sobrecarga para a equipe, riscos à segurança dos profissionais e pacientes e, consequentemente, maiores riscos de EA, comprometendo a qualidade dos serviços prestados. Nesse contexto, nota-se que a sobrecarga de trabalho aumenta a chance de riscos à segurança dos profissionais e pacientes, relacionados ao esforço físico da equipe de enfermagem, além de ocasionar risco de quedas dos pacientes; riscos para deslocamento de dispositivos; e riscos de infecção (Magalhães et al., 2015).

\section{Considerações Finais}

A revisão integrativa dos artigos selecionados possibilitou a análise da importância da segurança do paciente no cenário brasileiro, um dos temas mais discutidos na atualidade, como comprova a implantação, a nível nacional, dos Núcleos de Segurança do Paciente em todos os estabelecimentos de saúde, que inicialmente desenvolveram ações direcionadas aos gestores e profissionais da saúde. 
Os resultados deste estudo revelaram a importância de inserir a segurança do paciente como disciplina dentro das instituições de ensino a fïm de capacitar, o quanto antes, os futuros profissionais para que estes promovam a mitigação da ocorrência de eventos adversos (EA) na atenção à saúde.

Embora já exista uma lei que trata da inclusão da temática segurança do paciente nos cursos da área da saúde, percebese que muitas instituições de ensino ainda possuem fragilidades. Com isso, o estudo mostrou a relevância de o conteúdo ser abordado a nível médio e da graduação.

Para trabalhos futuros pretende-se levar este estudo às instituições de saúde e escolas técnicas com o objetivo de capacitar os profissionais em segurança do paciente. Ademais, outras pesquisas poderiam contribuir para a criação de um material didático como ferramenta de ensino-aprendizagem eficaz nessa área.

\section{Referências}

Baptista, P. C. P., Pustiglione, M., Almeida, M. C. dos S., Felli, V. E. A., Garzin, A. C. A., \& Melleiro, M. M. (2015). Saúde dos trabalhadores de enfermagem e a segurança do paciente: O olhar de gerentes de enfermagem. Revista da Escola de Enfermagem da USP, 49(spe), 122-128. 10.1590/S0080623420150000800017

Barbosa, M. H., Floriano, D. R., Oliveira, K. F. de, Nascimento, K. G. do, \& Ferreira, L. A. (2016). Clima de segurança do paciente em um hospital privado. Texto \& Contexto - Enfermagem, 25(3), e1460015. 10.1590/0104-07072016001460015

Cauduro, G. M. R., Magnago, T. S. B. de S., Andolhe, R., Lanes, T. C., \& Ongaro, J. D. (2017). Segurança do paciente na compreensão de estudantes da área da saúde. Revista Gaúcha de Enfermagem, 38(2), e64818. 10.1590/1983-1447.2017.02.64818

Cestari, V. R. F., Florêncio, R. S., Moreira, T. M. M., Pessoa, V. L. M. de P., Barbosa, I. V., Lima, F. E. T., \& Custódio, I. L. (2016). Competências do enfermeiro na promoção da saúde de indivíduos com cardiopatias crônicas. Revista Brasileira de Enfermagem, 69(6), 1195-1203. 10.1590/0034-7167-2016-0312

Conselho Federal de Enfermagem. (2018). Cofen lança selo de qualidade da Enfermagem. Recuperado de http://www.cofen.gov.br/cofen-lanca-selo-dequalidade-da enfermagem_64408.html

Françolin, L., Gabriel, C. S., Bernardes, A., Silva, A. E. B. de C., Brito, M. de F. P., \& Machado, J. P. (2015). Gerenciamento da segurança do paciente sob a ótica dos enfermeiros. Revista da Escola de Enfermagem da USP, 49(2), 0277-0283. 10.1590/S0080-623420150000200013

Gasparino, R. C., Bagne, B. M., Gastaldo, L. S., \& Dini, A. P. (2017). Percepção da enfermagem frente ao clima de segurança do paciente em instituições públicas e privadas. Revista Gaúcha de Enfermagem, 38(3), e68240. 10.1590/1983-1447.2017.03.68240

Gomes, A. T. L., Salvador, P. T. C. O., Rodrigues, C. C. F. M., Silva, M. F., Ferreira, L. L., Santos, V. E. P. (2017). A segurança do paciente nos caminhos percorridos pela enfermagem brasileira. Revista Brasileira de Enfernagem, 70(1),139-46. 10.1590/0034-7167-2015-0139

Magalhães, A. M. M. de, Moura, G. M. S. S. de, Pasin, S. S., Funcke, L. B., Pardal, B. M., \& Kreling, A. (2015). Processos de medicação, carga de trabalho e a segurança do paciente em unidades de internação. Revista da Escola de Enfermagem da USP, 49(spe), 43-50. 10.1590/S0080-623420150000700007

Marra, V. N.; Sette, M. L. (2016). Guia curricular de segurança do paciente da Organização Mundial da Saúde: Edição multiprofissional. Rio de Janeiro: Autografia. Disponível em: Recuperado de https://apps.who.int/iris/bitstream/handle/10665/44641/9788555268502-por.pdf;sequence=32

Möller, G., \& Magalhães, A. M. M. de. (2015). Banho no leito: Carga de trabalho da equipe de enfermagem e segurança do paciente. Texto \& Contexto Enfermagem, 24(4), 1044-1052. 10.1590/0104-0707201500003110014

Pinto, D. M. de, Schons, E. S., Busanello, J., \& Costa, V. Z. da. (2015). Segurança do paciente e a prevenção de lesões cutâneo-mucosas associadas aos dispositivos invasivos nas vias aéreas. Revista da Escola de Enfermagem da USP, 49(5), 775-782. 10.1590/S0080-623420150000500010

Raimondi, D. C., Bernal, S. C. Z., Souza, V. S. de, Oliveira, J. L. C. de, \& Matsuda, L. M. (2017). Higienização das mãos: Adesão da equipe de enfermagem de unidades de terapia intensiva pediátricas. Revista CUIDARTE, 8(3), 1839-1848. 10.15649/cuidarte.v8i3.437

Santiago, T. H. R., \& Turrini, R. N. T. (2015). Cultura e clima organizacional para segurança do paciente em Unidades de Terapia Intensiva. Revista da Escola de Enfermagem da USP, 49(spe), 123-130. doi: 10.1590/S0080-623420150000700018

Silva, A. T., Alves, M. G., Sanches, R. S., Terra, F. S., \& Resck, Z. M. R. (2016). Assistência de enfermagem e o enfoque da segurança do paciente no cenário brasileiro. Saúde em Debate, 40(111), 292-301. 10.1590/0103-1104201611123

Siman, A. G., \& Brito, M. J. M. (2016). Mudanças na prática de enfermagem para melhorar a segurança do paciente. Revista Gaúcha de Enfermagem, 37(spe), e68271. 10.1590/1983-1447.2016.esp.68271

Teixeira, J. P. D. de S., Rodrigues, M. C. S., Machado, V. B. (2012). Educação do paciente sobre regime terapêutico medicamentoso no processo de alta hospitalar: Uma revisão integrativa. Revista Gaúcha de Enfermagem, 33(2), 186-196. Recuperado de https://www.scielo.br/pdf/rgenf/v33n2/26.pdf

Urbanetto, J. S., \& Gerhardt, L. M. (2013). Segurança do paciente na tríade assistência ensino pesquisa. Revista Gaúcha de Enfermagem, 34(3), 8-9. Recuperado de https://seer.ufrgs.br/RevistaGauchadeEnfermagem/article/view/43294/27285 
Research, Society and Development, v. 10, n. 14, e348101422199, 2021

(CC BY 4.0) | ISSN 2525-3409 | DOI: http://dx.doi.org/10.33448/rsd-v10i14.22199

Wegner, W., Silva, S. C. da, Kantorski, K. J. C., Predebon, C. M., Sanches, M. O., \& Pedro, E. N. R. (2016). Educação para cultura da segurança do paciente: Implicações para a formação profissional. Escola Anna Nery, 20(3), e20160068. 10.5935/1414-8145.20160068

Yoshikawa, J. M., Sousa, B. E. C. de, Peterlini, M. A. S., Kusahara, D. M., Pedreira, M. da L. G., \& Avelar, A. F. M. (2013). Compreensão de alunos de cursos de graduação em enfermagem e medicina sobre segurança do paciente. Acta Paulista de Enfermagem, 26(1), 21-29. 10.1590/S0103-21002013000100005 